\title{
Psicooncología
}

ISSN: 1696-7240

\section{Intervenciones psicológicas eficaces en pacientes con cáncer de mama en Latinoamérica y España: una revisión sistemática}

\author{
María Fernanda Quintero, ${ }^{1, *}$; Carolyn Finck ${ }^{2}$
}

Recibido: 30 de diciembre de 2017 / Aceptado: 24 de febrero de 2018

Resumen. Objetivo: Este estudio es una revisión sistemática que buscó identificar la evidencia disponible en la literatura científica de Latinoamérica y España sobre las intervenciones psicológicas eficaces en mujeres con cáncer de mama, entre el 2006 y 2016. Método: Las bases de datos empleadas fueron BVS y PsycNet-APA, utilizando los descriptores: cáncer de mama, intervenciones psicológicas, psicooncología, y psicoterapia, tanto en inglés como en español. También se realizó la búsqueda en Google Académico, con la combinación "Cáncer de mama and intervenciones psicológicas". Resultados: Se encontraron 17 artículos en los que el modelo teórico más implementado fue el modelo cognitivo conductual, seguido por el modelo de intervenciones grupales y psicosociales. Sin embargo, también se encontraron otros modelos menos comunes para esta población. Así mismo se identificaron limitaciones en términos de metodología en estas investigaciones, lo que dificulta la generalización de sus resultados. Conclusiones: Se considera que es necesario la realización de investigaciones con diseños robustos en Latinoamérica y España sobre intervenciones psicooncológicas eficaces en pacientes con cáncer de mama.

Palabras claves: Intervenciones psicológicas; cáncer de mama; Latino-américa.

\section{[en] Effective psychological intervention for breast cancer patients in Latin} America and Spain: A systematic review

\begin{abstract}
Objective: The aim of this systematic review was to identify the evidence available in the Latin America and Spain scientific literature about the effective psychological intervention for breast cancer, between 2006 and 2016. Method: The databases included were BVS and PsycNetAPA, using the following descriptors: breast cancer, psychological interventions, psychooncology, and psychotherapy, in English and Spanish. The search was also done in Google Academics with the descriptors: "Cáncer de mama and intervenciones psicológicas", in Spanish. Results: Only 17 articles were found that met our inclusion criteria and showed the evidence of psychological intervention for breast cancer patient in Latin América and Spain between 2006 and 2016. The theoretical model most frequently applied was CBT, followed by other types of psychosocial intervention. Nevertheless, other novel models to these population were also found. Limits in the methodology of these investigation were identified, which hinders the generalization of results. Conclusions: More research with according methodology design to prove the efficacy of a psychological intervention for breast cancer patient, is very much needed in Latin America and Spain.
\end{abstract}

Keywords: Psychological Intervention; breast cancer; latin america.

1 María Fernanda Quintero. Departamento de Psicología. Universidad de los Andes. Bogota, Colombia.

E-mail: mf.quintero12@uniandes.edu.co

2 Carolyn Finck, Departamento de Psicología. Universidad de los Andes, Bogotá. Colombia.

E-mail: cfinck@uniandes.edu.co

* Dirección de correspondencia: María Fernanda Quintero Marzola, Departamento de Psicología. Universidad de los Andes. Bogota, Colombia. E-mail: mf.quintero12@uniandes.edu.co 
Sumario. 1. Introducción 1.1. Intervenciones psicológicas para las respuestas emocionales del cáncer 2. Metodología 2.1. Procedimiento 2.2. Criterios de inclusión 2.3. Criterios de exclusión 3. Resultados 3.1. Diseño y publicación 3.2 Resultados de las investigaciones 3.3 Información sociodemográfica de las muestras 4. Discusión 5. Conclusiones y recomendaciones 5.1. Limitaciones de este estudio 5.2. Futuras investigaciones 6. Agradecimientos 7. Referencias bibliográficas.

Cómo citar: Quintero MF, Finck C. Intervenciones psicológicas eficaces en pacientes con cáncer de mama en Latinoamérica y España: una revisión sistemática. Psicooncología 2018;15:49-64. Doi: 10.5209/PSIC.59174.

\section{Introducción}

El cáncer ocupa el segundo lugar entre las principales causas de muerte en el mundo, después de las enfermedades cardiovasculares ${ }^{(1)}$. Uno de los principales tipos de cáncer que afecta a la mujer es el mamario, ocasionando 521.000 defunciones en 2012 a nivel mundial ${ }^{(2)}$ Latinoamérica tiene aproximadamente el $10 \%$ de la incidencia y mortalidad del cáncer de mama a nivel mundial ${ }^{(3-6)}$. Uno de los rasgos característicos del cáncer, además de sus complicaciones físicas, es el impacto psicológico que ocasiona debido a la experiencia de una continua incertidumbre en medio de una crisis que amenaza la vida ${ }^{(6)}$. Diferentes estudios han encontrado que las personas con cáncer experimentan emociones como: angustia, ansiedad, depresión, pensamientos intrusivos y de evitación, además de sintomatología de estrés postraumático ${ }^{(7-9)}$. Todo lo anterior como respuesta a características de la enfermedad tales como el dolor, la invasión, la posibilidad de muerte o los efectos secundarios de los tratamientos ${ }^{(9)}$. Dicha experiencia emocional se presenta en cáncer de mama debido a que el diagnóstico se asocia, adicionalmente en muchos casos, con disfunción sexual, trastornos de la imagen corporal, y evitación social ${ }^{(10)}$.

\subsection{Intervenciones psicológicas para las respuestas emocionales del cáncer}

La $\mathrm{APA}^{(11)}$, sugiere que se debe brindar un apoyo psicológico tanto individual como grupal, en los casos de cáncer de mama; el primero para modificar patrones de pensamiento y conducta, y el segundo para recibir apoyo emocional de mujeres que estén enfrentando la misma situación. De esta manera Robles (2010) ${ }^{(12)}$; Monsalve, Gómez-Carretero y Soriano (2006) ${ }^{(13)}$, Bragado (2009)(14); Salas et al., (2003) ${ }^{(15)}$; Bellver y Moreno (2009) ${ }^{(16)}$; resaltan el modelo de intervención cognitivo-conductual. Según estos autores, dicho modelo es funcional para el tratamiento de individuos con hábitos y pensamientos nocivos para la salud. Su aplicación en el contexto de los pacientes con cáncer se centra en el alivio de los síntomas a través de la relación existente entre cognición, afecto y conducta. Por su parte, Robles $(2010)^{(12)}$ y Pietro $(2004)^{(17)}$ mencionan la terapia cognitiva como un fuerte tipo de intervención para los pacientes con cáncer.

De la misma manera, a continuación, se proponen otros tipos de intervenciones las cuales también han mostrado eficacia en esta población. Matsuda et al. (2014) ${ }^{(18)}$ y Font y Rodriguez $(2007)^{(19)}$ resaltan los resultados de las intervenciones psicosociales en la mejoría de la sintomatología de cáncer de mama, y las intervenciones psicoeducativas para mejorar la experiencia emocional durante la enfermedad, que tienen como efecto 
aumentar la calidad de vida, y adaptación a la patología. Asimismo, McGregor y Antoni $(2008)^{(20)}$, sostienen que las intervenciones psicosociales promueven la adaptación psicológica en personas que sufren de cáncer de mama y su tratamiento, al igual que modulan el funcionamiento de la respuesta proliferativa de linfocitos. Kangas, Bovbjerg, y Montgomery $(2008)^{(21)}$ sostienen que las intervenciones relacionadas con la terapia expresiva, así como las de componentes cognitivo-conductuales, ayudan a reducir la fatiga en personas con cáncer. Este último tipo de intervención, según los anteriores autores, ayuda a aumentar la vitalidad en estos pacientes, así como otras intervenciones relacionadas con psicoeducación. De igual manera McGregor y Antoni $(2008)^{(20)}$, mencionan que se encontraron resultados positivos de la terapia cognitivo conductual en los niveles de cortisol tardíos en mujeres con cáncer de mama no metastásico. Por otro lado, Smith et al. $(2005)^{(22)}$, encontraron que el mindfulness tiene efectos positivos que se mantienen en el tiempo, sobre la reducción de estrés, mejoramiento en la calidad de vida, y de la calidad del sueño. Igualmente, CasellasGrau, Font y Vives (2014) ${ }^{(23)}$, encontraron que las terapias positivas ayudan a mejorar la calidad de vida, bienestar, esperanza, felicidad, optimismo, y satisfacción de la vida, en mujeres con cáncer de mama.

El presente estudio tiene como objetivo realizar una búsqueda sistemática de los resultados de la investigación publicados en Latinoamérica y España, entre 2006 y 2016 sobre las intervenciones psicológicas eficaces en pacientes con cáncer de mama, con el fin de identificar el estado de la investigación en la región, así como para observar las diferencias y similitudes con los resultados en otras poblaciones del mundo.-

\section{Metodología}

\subsection{Procedimiento}

La búsqueda se realizó en el 2016, en las bases de datos BVS y PsycNet APA. De igual manera se utilizó el buscador de Google Académico con el objetivo de obtener resultados más amplios. Para dar respuesta a la pregunta planteada en esta investigación, se tuvieron en cuenta los "Descriptores en Ciencia de la Salud (DeCS)" de "cáncer de mama" y "psicología". También se hizo una búsqueda con los términos "Protocolos psicológicos", "Terapia Psicológica", "Intervención Psicológica" y "Psicooncología/Psico-oncología" sin encontrar resultados. Finalmente, la búsqueda sistemática se realizó utilizando las combinaciones de descriptores "cáncer de mama and intervenciones psicológicas", "cáncer de mama and psicooncología”, y, "cáncer de mama and psicoterapia", en español; y "breast cancer and psychological interventions", "breast cancer and psychooncology", "breast cancer and psychoterapy", en inglés. En la búsqueda en Google Académico, se utilizó la combinación "Cáncer de mama and intervenciones psicológicas", en español.

La revisión inicial se realizó sólo de títulos y abstracts. De esta manera fueron seleccionadas aquellas investigaciones que cumplían los criterios que se expondrán a continuación, los cuales fueron organizados utilizando el programa de referencias bibliográficas ${ }^{\circledR}$ Mendely reference manager. Posteriormente se realizó la revisión de duplicados y luego se hizo una revisión adicional de cada uno de los artículos que resultaron, descartando aquellos que no cumplían los criterios de inclusión en el estudio. 


\subsection{Criterios de inclusión}

Durante la búsqueda, se tuvieron en cuenta publicaciones con población de cáncer de mama, inicialmente en Latinoamérica, que mostraran la evidencia científica sobre las intervenciones psicológicas eficaces en esta población, sin importar el modelo teórico y las técnicas utilizadas. Así mismo, se incluyeron únicamente investigaciones de carácter cuantitativo, con un modelo experimental (con o sin grupo control) y que fueran explícitos en las técnicas aplicadas y las variables a intervenir en estas pacientes. En una primera búsqueda se tuvieron en cuenta investigaciones realizadas desde el año 2010 en Latinoamérica, sin embargo, tras los pocos resultados obtenidos, se decidió ampliar el rango de búsqueda al año 2006, y se incluyeron en esta segunda búsqueda estudios realizados en España.

\subsection{Criterios de exclusión}

Las investigaciones tenidas en cuenta para este estudio no debían ser de otra población diferente a pacientes con cáncer de mama. Adicionalmente, debían ser originales de Latinoamérica y España, y los protocolos aplicados debían proceder de la psicología. No se tendrían en cuenta aquellos estudios con técnicas provenientes del arte, religión, etc. También se excluyeron investigaciones y estudios cualitativos, revisiones bibliográficas, análisis de casos, revisiones sistemáticas y meta análisis.

\section{Resultados}

En la primera búsqueda a través de las ecuaciones anteriormente descritas, se obtuvo como resultado un total de 3.617 artículos, de los cuales se revisaron 1.161 acorde a los filtros relacionados con países (Latinoamérica -incluyendo Brasil) y año (20102016). De igual manera la búsqueda en Google Académico dio como resultado 212 artículos que fueron revisados adicionalmente. De esta forma se revisaron un total de 1.373 artículos (ver figura 1).

En la segunda búsqueda en la que se amplió el año de publicación se obtuvo un total de 5.072 resultados, de los cuales se revisaron 942, utilizando los filtros correspondientes a países y año (2006-2010). Por su parte, la búsqueda en Google Académico arrojó 221 artículos que también fueron revisados. Se revisaron 1.163 artículos en total (ver figura 2). En general, se revisaron 2.536 artículos que resultaron de las dos búsquedas.

Posterior al proceso de revisión, se obtuvieron sólo seis artículos en la primera búsqueda que correspondían con los criterios de inclusión, y nueve en la segunda. Es así como, teniendo en cuenta todo el proceso de búsqueda, se encontraron en su totalidad diecisiete artículos $^{(24-40)}$ que reflejan la evidencia científica publicada en Latinoamérica y España sobre las intervenciones psicológicas eficaces en pacientes con cáncer de mama desde 2006. 
Figura 1. Diagrama de flujo de la primera búsqueda

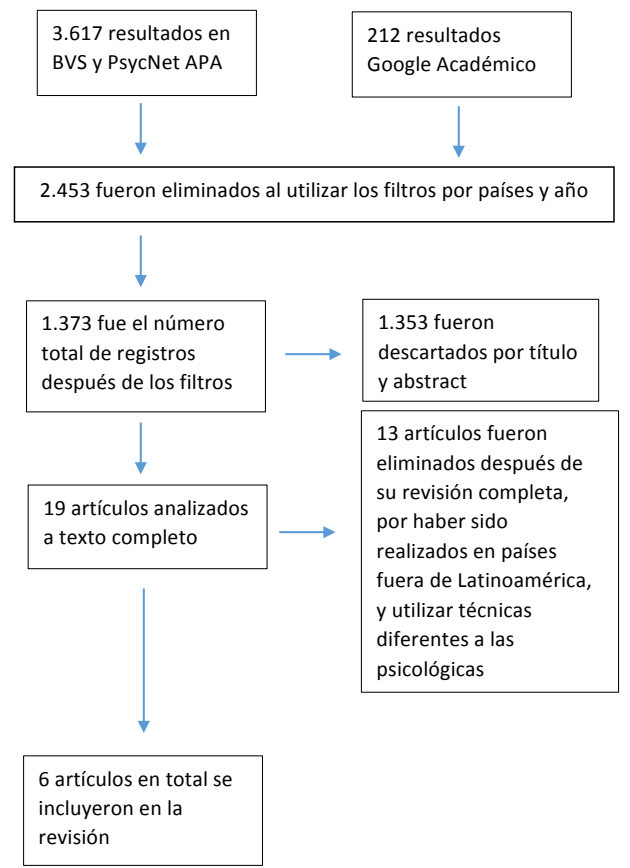

Figura 2. Diagrama de flujo de la segunda búsqueda

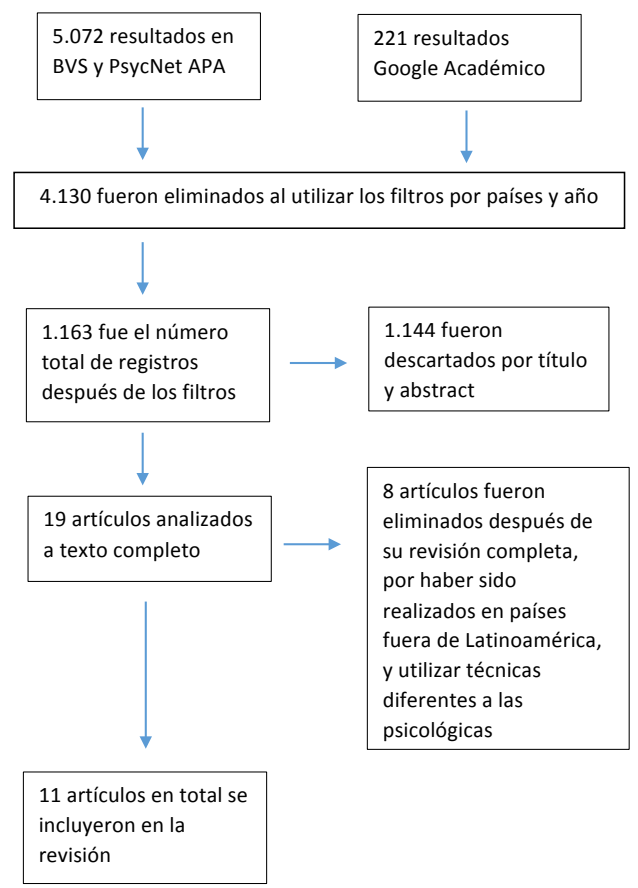




\subsection{Diseño y publicación}

Los artículos encontrados en esta búsqueda sistemática estudiaron diferentes variables dependientes. De los diecisiete artículos, ocho estudiaron como variables dependientes estados emocionales y cognición ${ }^{(25,27,28,33,35,36,39,40)}$. Otros tres, estudiaron variables dependientes relacionadas sólo con estados emocionales $^{(31,34,38)}$. Dos estudios diferentes, incluyeron variables dependientes relacionadas con estados emocionales y conducta ${ }^{(26,29)}$. Sólo uno incluyó como variables dependientes aspectos emocionales y físicos ${ }^{(24)}$; otro artículo, variables emocionales, conductuales y cognitivas ${ }^{(32)}$; uno más, sólo variables conductuales ${ }^{(37)}$, y otro, sólo variables cognitivas ${ }^{(30)}$ (ver figura 3 ).

Por otro lado, en la figura 4, se podrá observar el tipo de metodología utilizada en cuanto a la variable independiente y grupos de análisis utilizados. Ocho de las

Figura 3. Variables dependientes de los artículos

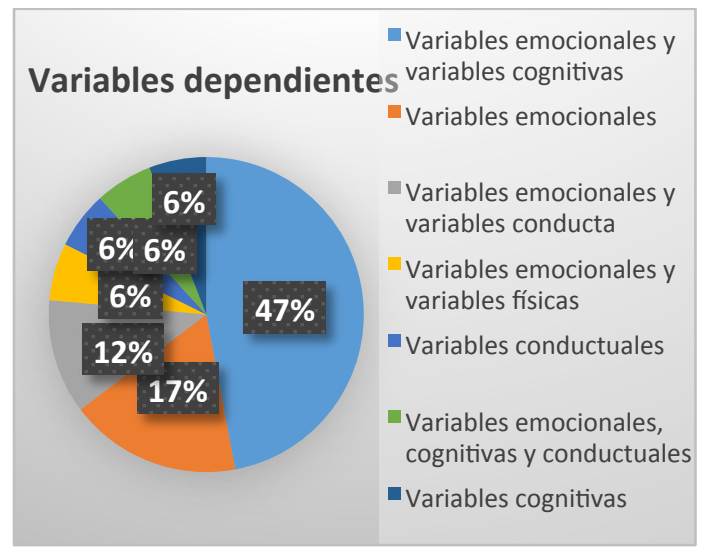

Figura 4. Metodología implementada en los artículos

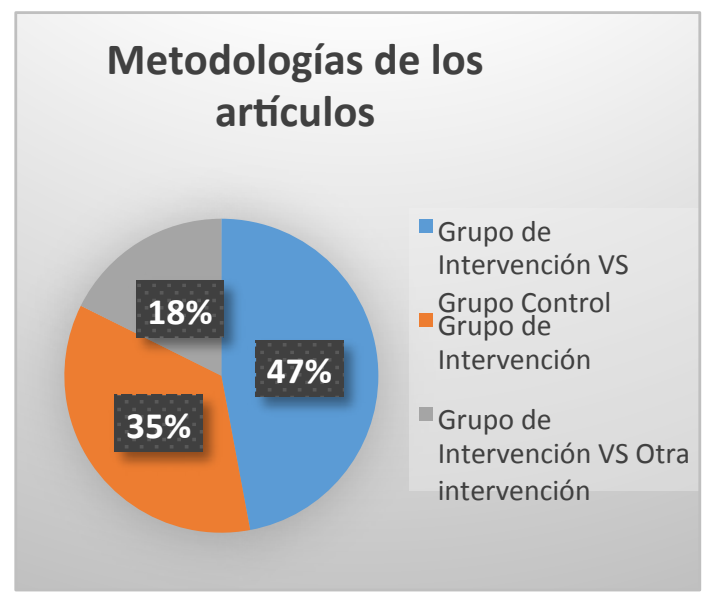


investigaciones tenidas en cuenta realizaron análisis de sus resultados comparando grupo experimental (intervención) con grupo control ${ }^{(24,26,28,29,33,35,36,40)}$; seis de ellos sólo utilizaron grupo de intervención ${ }^{(25,30-32,34,38)}$; y otros tres realizaron análisis entre dos grupos realizando intervenciones en ambos ${ }^{(27,37,39)}$.

Así mismo, se observó que los cuestionarios y test más aplicados para medir las anteriores variables fueron: la Escala de Depresión y Ansiedad Hospitalaria (HADS), utilizada en cinco artículos ${ }^{(27,29,32,38,39)}$; y, el Inventario Ansiedad EstadoRasgo (STAI) ${ }^{(24,26,28,34)}$, la escala de Autoestima de Rosenberg ${ }^{(28,33,35,40)}$ y el Inventario de Depresión de Beck (BDI) ${ }^{(24,26,28,33)}$, los cuales fueron utilizados en cuatro artículos respectivamente.

Por otro lado, la metodología de diez de las investigaciones ${ }^{(24,26,28,31,32,34-36,38,40)}$ estaba basada en medidas pre y post; otras cinco realizaron, además, medidas de seguimiento ${ }^{(27,29,30,33,39)} ; \mathrm{y}$, dos más tomaron datos durante la intervención, adicional al pre y post ${ }^{(25,37)}$.

Adicionalmente, teniendo en cuenta la clasificación mencionado por Glanz y Bishop (2010) ${ }^{(41)}$, del grado de involucramiento del modelo de intervención en el estudio, se identificó que tres artículos tenían un nivel de involucramiento " $a$ ", denominado "Informado por la Teoría"(25,26,31), en donde se identifica el modelo teórico, pero este no fue utilizado en los componentes específicos del estudio y medidas. Seis de ellos, se clasificaron en un nivel " $b$ ", nombrado como "Teoría Aplicada"(28,30,33,34,36,38), en donde el marco teórico es especificado y varios de los constructos son aplicados y hacen parte del estudio. Otros seis artículos, se incluyeron en el nivel "c", denominado "Teoría Probada"(27,29,32,35,37,39), donde se especifica el modelo teórico y más de la mitad de los constructos de este son incluidos y evaluados en el estudio; así mismo que se comparen dos o más teorías en la investigación. Finalmente, un estudio, se clasificó en el nivel "d", llamado "Creando Teoría"(40), en donde se revisa una nueva teoría y se desarrollan constructos específicos, medidas y análisis en el estudio. Por último, solo uno de los artículos no pudo ser clasificado en ningún nivel debido a que no especificó modelo teórico ${ }^{(24)}$.

En cuanto a los modelos teóricos reconocidos y utilizados en los artículos seleccionados y sus intervenciones se encuentran: Modelo Cognitivo Conductual (CBT) $)^{(27,28,31,32,38,39)}$, Terapias de Aceptación y Compromiso (ACT) ${ }^{(27,29)}$, Eye Movement Desensitizacion Process (EMDP) ${ }^{(34)}$, Modelo Narrativo ${ }^{(33)}$, Psicología positiva ${ }^{(35)}$, Psicoterapia integradora ${ }^{(36)}$, RIME (relaxation, mental images, and spirituality) ${ }^{(40)}$, hipnoterapia $^{(37)}$, e intervenciones grupales y psicosociales ${ }^{(25,26,28,30,38,39)}$ (ver figura 5). Como se mencionó anteriormente, uno de ellos no es explícito en el modelo de intervención que utiliza, sin embargo, sus técnicas son basadas en el modelo $\mathrm{CBT}^{(24)}$.

Por otra parte, las técnicas más empleadas utilizadas en más de tres artículos, fueron: relajación con imaginación guiada ${ }^{(24,26,32,37-40)}$, relajación muscular progresiva $^{(24,27,28,32,38,39)}$; seguidas por: relajación con respiración ${ }^{(27,28,39)}$, detención del pensamiento ${ }^{(27,32,38,39)}$, distracción cognitiva ${ }^{(32,38,39)}$, distorsión cognitiva ${ }^{(27,36,39)}$, entrenamiento en comunicación ${ }^{(30,35,39)}$, entrenamiento en planeación de actividades placenteras $^{(28,35,39)}$, y entrenamiento en autoinstrucciones ${ }^{(27,28,39)}$.

Por otro lado, los artículos en su mayoría fueron publicados en la revista Psicooncología ${ }^{(25-29,31,32,37-39)}$, siendo también la mayor parte de ellos publicados en español ${ }^{(25-28,31-34,36-39)}$, y provenientes de España ${ }^{(25,27-30,35,36,38,39)}$. Los otros países de Latinoamérica donde se realizaron investigaciones (ver figura 6) que también muestran evidencia científica de las intervenciones psicológicas realizadas en 
Figura 5. Modelos de intervención reconocidos y empleados en los artículos incluidos en el estudio

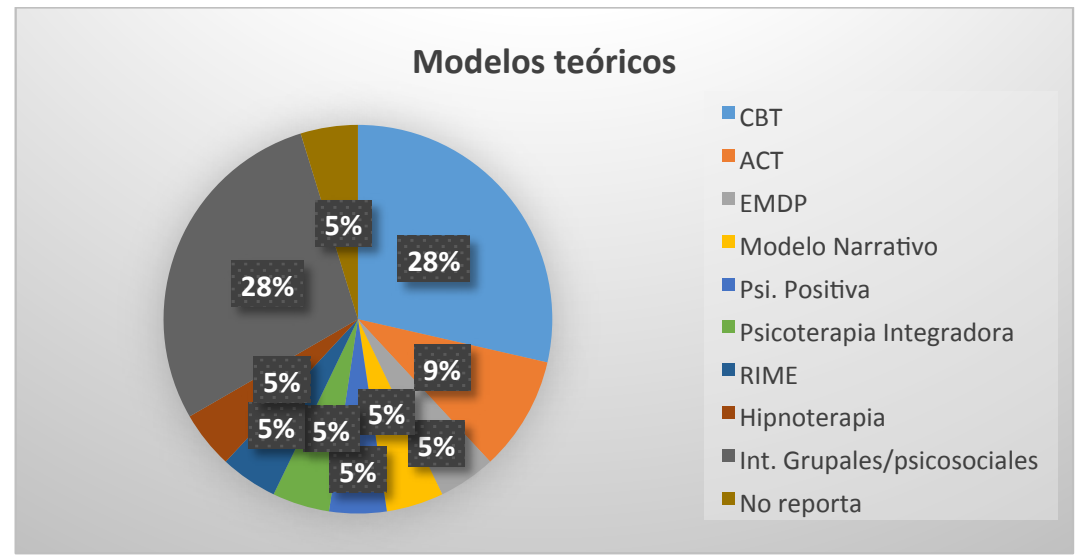

pacientes con cáncer de mama entre 2006 y 2016 fueron: México ${ }^{(31,32,37)}$, Brasil ${ }^{(24,40)}$, $\mathrm{Cuba}^{(34)}$, Chile ${ }^{(33)}$ y Venezuela ${ }^{(26)}$. No se encontraron artículos para el resto de países latinoamericanos.

\subsection{Resultados de las investigaciones}

Los resultados de las investigaciones varían según modelo, y variables estudiadas. A continuación, se mostrarán algunos resultados según las variables de estudio.

En las investigaciones en las que se trabajó con variables emocionales ${ }^{(24-36,38,39)}$, se reportan resultados positivos relacionados con la disminución del malestar y estado de ánimo tanto negativo como bajo, así como sintomatología postraumática, después de la intervención. En algunas de las investigaciones se observa una continuidad de estos resultados en sus medidas de seguimiento ${ }^{(27,29,30,33,39)}$. En el caso de Telles

Figura 6. Países de las publicaciones tenidas en cuenta en este estudio

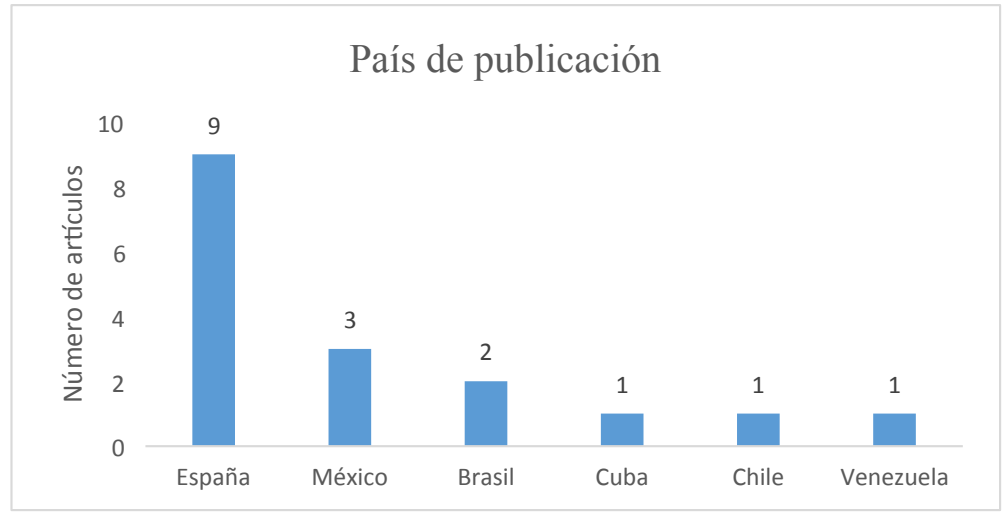


et al. (2007) $)^{(24)}$, reportan un tamaño del efecto medio en el grupo experimental en las variables: ansiedad, y, ansiedad rasgo y estado. De igual manera, González, González y Estrada (2015)(32), informaron un efecto de alta magnitud en depresión y salud psicológica. Montesinos y Luciano (2016) ${ }^{(29)}$, reportan un efecto significativo en ambas medidas de seguimiento, relacionado con las variables intensidad e interferencia del estrés crónico, ansiedad e hipocondría.

Los resultados de investigaciones cuyas variables estaban relacionadas con los procesos cognitivos, muestran una mejoría significativa sobre la percepción de la calidad de vida, así como de autoestima ${ }^{(24,25,28,30,32,35,36,40)}$. En este caso Telles et al. $(2007)^{(24)}$, reportan un tamaño del efecto medio en calidad de vida.

De igual manera, en los resultados relacionados con variables dependientes de tipo conductual, se identifica una disminución en comportamientos interferentes y consecuentes con la enfermedad, y un aumento en aquellos que facilitan el afrontamiento a esta última ${ }^{(26,29,32,37)}$. Bernal et al. $(2015)^{(37)}$ reportan el tamaño del efecto en valores negativos mayores a 0,8 , relacionado con latencia del sueño, duración, eficacia, y calidad del sueño.

Por último, los resultados en las variables físicas permiten concluir que no se encontraron diferencias en las medidas de cortisol ni en la proliferación de células T, posteriores a la intervención ${ }^{(24)}$.

De igual manera, los modelos teóricos utilizados también presentan resultados que parecen ser positivos y por lo cual muestran su eficacia en mejorar las condiciones de las mujeres con cáncer de mamá, teniendo en cuenta las variables escogidas en los estudios correspondientes, y sus técnicas. A continuación, se relacionarán los modelos y resultados respectivos.

Aquellas investigaciones que utilizaron estrategias derivadas del modelo cognitivo

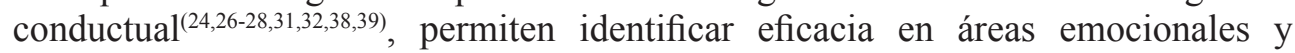
percepción de calidad de vida. Por su parte, las derivadas de $\mathrm{ACT}^{(27,29)}$ permiten observar el funcionamiento de este modelo en áreas emocionales y comportamentales. Así mismo, los resultados propuestos por investigaciones relacionadas con los modelos $\operatorname{EMDP}^{(34)}$, Intervención Psicosocial( ${ }^{(25,26,28,30,38,39)}$, Terapia Narrativa ${ }^{(33)}$, Psicología positiva ${ }^{(35)}$, Psicoterapia integradora ${ }^{(36)}$, y RIME $^{(40)}$, mostraron resultados positivos en el área afectiva. Por último, el modelo relacionado con la hipnoterapia ${ }^{(37)}$ reflejó buenos resultados en el área comportamental.

\subsection{Información sociodemográfica de las muestras}

En cuanto a las características sociodemográficas de las poblaciones estudiadas, las características de la muestra varían en algunos artículos. Las edades de estas pacientes oscilaban entre 18 y 79 años, y en la totalidad de los artículos reportaban que la mayoría de sus participantes estaban casadas. Cinco de ellos reportaron que el mayor número de participantes tenían hijos ${ }^{(25,32,33,35,37)}$, y diez no reportaron información acerca de la maternidad ${ }^{(24,27-31,36,38-40)}$.

De igual manera y en cuanto al nivel educativo, cinco artículos reportaron que la mayoría de sus participantes tenían educación primaria ${ }^{(25,31-33,35)}$, otro mencionó que la mayor parte de las mujeres tenían educación superior ${ }^{(36)}$, y otro más afirmó que las pacientes en su mayoría eran Bachilleres ${ }^{(30)}$. Araújo et al. $(2015)^{(40)}$, por su parte, a diferencia de los demás artículos, reporta diferencias en la escolaridad en los grupos de 
estudio, siendo la mayoría del grupo control, primaria; y la del grupo de intervención, secundaria. Otros siete artículos, no reflejan información de la escolaridad ${ }^{(24,27-29,37-39)}$.

En cuanto a la ocupación de las participantes, cuatro artículos confirmaron tener en sus grupos amas de casa en su mayoría ${ }^{(30-32,38)}$; otros dos, reportaron que la mayoría eran empleadas $^{(24,35)}$; y nueve, no reportan ocupación de sus participantes ${ }^{(25,27-29,33,36,37,39,40)}$. Por último, dos de los artículos, no reportaban datos sociodemográficos ${ }^{(26,34)}$.

Es importante resaltar que el único artículo que reportó datos de estratificación socioeconómica, fue Bernal et al. (2015) $)^{(37)}$, el cual refirió una condición económica baja en el grupo control, y alta en el experimental.

Así mismo, la distribución de la condición de la enfermedad de las pacientes varía también entre algunos artículos. Cinco investigaciones no tuvieron criterios de exclusión ni inclusión en esta área ${ }^{(26,27,29,32,35)}$; tres tomaron como muestra mujeres libres de la patología posterior a tratamiento ${ }^{(25,28,39)}$; y otros tres tomaron como muestra, mujeres mastectomizadas ${ }^{(30,36,40)}$.

\section{Discusión}

Esta revisión sistemática es la primera, según nuestro conocimiento, que se realiza buscando evidencia sobre las intervenciones psicológicas que se realizan en Latinoamérica y España, con pacientes con cáncer de mama. El número de artículos incluidos en este estudio no difiere de los encontrados en otras revisiones a nivel mundial $^{(18-20,23)}$. Sin embargo, al sumar toda la evidencia que se establece globalmente, se considera que es relativamente poca teniendo en cuenta que el cáncer de mama es una enfermedad en crecimiento y una de las principales que afecta a las mujeres alrededor del mundo(2).

En cuanto a los modelos de las intervenciones, y técnicas aplicadas en los artículos, se observa que la mayoría provienen del modelo teórico cognitivo-conductual ${ }^{(24,26-28,31}$, 32,38,39), el cual es uno de los modelos más empleados en el sector salud ya que sus objetivos buscan aliviar los síntomas realizando una intervención integral que influya los componentes cognitivo-afectivo-comportamentales ${ }^{(12-16,20,21)}$, los cuales finalmente son los componentes de la experiencia emocional ${ }^{(42)}$. Igualmente, las técnicas más utilizadas provienen de este modelo teórico, destacándose técnicas como: relajación con imaginación guiada(24,26,32,37-40), relajación muscular progresiva ${ }^{(24,27,28,32,38,39)}$; relajación con respiración ${ }^{(27,28,39)}$, detención del pensamiento ${ }^{(27,32,38,39)}$,distracción $^{2}$ cognitiva ${ }^{(32,38,39)}$, distorsión cognitiva ${ }^{(27,36,39)}$, entrenamiento en comunicación ${ }^{(30,35,39)}$, entrenamiento en planeación de actividades placenteras ${ }^{(28,35,39)}$, y entrenamiento en autoinstrucciones $^{(27,28,39)}$.

Teniendo en cuenta la evidencia a nivel mundial sobre las intervenciones psicológicas eficaces en pacientes con cáncer y cáncer de mama ${ }^{(18-23)}$, en Latinoamérica y España al parecer sólo se están realizando estudios con intervenciones promisorias según la evidencia mundial, al aplicar los modelos: psicosociales ${ }^{(25,26,28,30,38,39)}$, $\mathrm{CBT}^{(27,28,31,32,38,39)}$ y psicología positiva ${ }^{(35)}$. El resto de la evidencia científica se está realizando con intervenciones basadas en modelos de los cuales aún no se reporta eficacia a nivel mundial en pacientes con esta patología, como lo son: RIME ${ }^{(40)}$, $\mathrm{ACT}^{(27,29)}, \mathrm{EMDP}^{(34)}$, Hipnoterapia ${ }^{(37)}$, terapia integradora ${ }^{(36)}$, y terapia narrativa ${ }^{(33)}$.

Lo anterior permite observar que, aunque la mayoría de los estudios encontrados $^{(26-28,30-32,35,38,39)}$ son coherentes con los modelos que muestran evidencia 
en esta población a nivel mundial (teniendo en cuenta los resultados encontrados en diferentes metaanálisis y búsquedas sistemáticas, incluyendo literatura en inglés $\left.{ }^{(18-23)}\right)$, también se están realizando estudios que prueban la eficacia de otros, no tenidos en cuenta para esta patología en la literatura global ${ }^{(27,29,33,34,36,37,40)}$. Por lo tanto, es interesante señalar que en Latinoamérica y España se está experimentando con modelos distintos a la evidencia que se muestra a nivel mundial. Así mismo, es importante resaltar que teniendo en cuenta lo anterior y el grado de involucramiento del modelo de intervención en el estudio ${ }^{(41)}$, se encuentra un gran número de artículos aplicando teoría ${ }^{(28,30,33,34,36,38)}$, y probando teoría ${ }^{(27,29,32,35,37,39)}$.

Por otro lado, la muestra utilizada en los artículos incluidos en este estudio varía con respecto a los diferentes estados de la patología, así como también se diferencian en la edad en las que se realizaron las intervenciones. Sin embargo, la mayoría de ellos reportaba que la gran parte de sus pacientes eran casadas ${ }^{(24,25,27-33,35-40)}$, lo cual permite discutir si los resultados de estas intervenciones hubieran sido los mismos en mujeres que no estuvieran recibiendo el apoyo de un conyugue, o con poco apoyo social. Adicionalmente, teniendo en cuenta que la muestra de las diferentes investigaciones difiere la una de la otra en cuanto al estadio de la patología, parecería que las intervenciones psicológicas pueden ser aplicadas en diferentes momentos de la enfermedad, así como en diferentes edades, mostrando resultados positivos.

Adicionalmente, también se identifica que la mayoría de los estudios incluidos ${ }^{(24-36,38,39)}$ estudiaron el impacto de las intervenciones en los estados emocionales de las pacientes. Sin embargo, estas intervenciones no se traducen necesariamente en una mejoría en la salud física, sino en aspectos psicológicos y bienestar general. En cuanto a la ansiedad estado-rasgo, Pocino et al., (2007) ${ }^{(26)}$ encontraron diferencias significativas en la ansiedad estado posterior al tratamiento, a diferencia de la ansiedad rasgo.

Por otro lado, sorprendentemente, en los artículos revisados hubo investigaciones que utilizaron como metodología de estudio sólo un grupo experimental, sin comparar los resultados con un grupo control para probar la funcionalidad de la intervención ${ }^{(25,30-32,34,38)}$. Y aunque no fue la mayoría, esta metodología de investigación hace más difícil generalizar y validar los resultados. Así mismo, llama la atención que

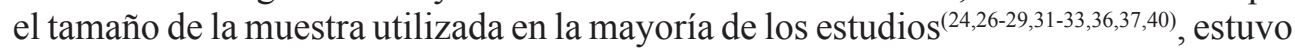
caracterizada por ser un número pequeño, lo cual también dificulta la generalización de los resultados. De esta manera se hace importante analizar los resultados de las intervenciones con precaución. De igual forma, que la mayor parte de los estudios incluidos en esta revisión hayan utilizado una medición de sólo pre y post ${ }^{24,26,28}$, $31,32,34-36,38,40)$ no permite observar si los resultados se mantienen en el tiempo tras la intervención. Sólo cinco estudios realizaron medidas de seguimiento ${ }^{(27,29,30,33,39)}$, donde sólo dos cumplieron con el tiempo mínimo requerido de espera para realizar medidas de seguimiento objetivas (6 meses) $)^{(27,30)}$.

\section{Conclusiones y recomendaciones}

Las limitaciones de los estudios escogidos para esta investigación se relacionan con cinco aspectos, algunas ya mencionadas: tamaño de la muestra, muestreo, metodología utilizada, medición, y reporte de tamaño del efecto de la intervención. 
Se consideró que el tamaño de la muestra en sólo seis artículos ${ }^{(25,30,34,35,38,39)}$ responde de manera adecuada a la representación de la población, teniendo en cuenta la metodología de investigación escogida y las variables que se querían estudiar; mientras que los demás ${ }^{(24,26-29,31-33,36,37,40)}$, se considera que incurrieron en error tipo I, en donde la muestra no parece ser representativa de la población, por lo tanto, se dificulta la generalización de los resultados. De la misma manera, el muestreo por conveniencia realizado por diferentes artículos ${ }^{(26,32-34,36,37)}$, influye en que los resultados obtenidos sean difíciles de generalizar.

Adicionalmente, el tipo de mediciones utilizada de la mayoría de los $\operatorname{artículos}^{(24-26,28,31,32,34-36,38,40)}$ en donde no se tuvo en cuenta tomarmedidas de seguimiento posterior a la intervención, no permite identificar si los hallazgos encontrados en cada una de las investigaciones se mantienen en el tiempo. Lo anterior es básico para identificar la funcionalidad de estas intervenciones en mujeres con cáncer de mama, ya que las dificultades de esta patología se pueden seguir mostrando posterior a que se finaliza el tratamiento.

De igual manera, la metodología escogida por algunos artículos en los que no hay grupo control ${ }^{(25,30-32,34,38)}$, también se considera una limitación ya que no es posible realizar comparaciones con algún otro grupo en donde no se realiza la misma intervención, por lo que la evaluación de la efectividad de esta última se ve afectada.

Enelmismosentido, otralimitantees quelamayoríadelos estudios ${ }^{(25-28,30,31,33,34,36,38-40)}$, no informan en su análisis estadístico el tamaño del efecto de su intervención $(d$ de Cohen), lo que es importante en términos cuantitativos para demostrar la efectividad de la misma en esta población.

Adicionalmente, es interesante que la mayoría de los artículos incluidos en esta investigación, tienen un grado de involucramiento del modelo de intervención en el estudio(41), relacionado con "Teoría Aplicada"(28,30,33,34,36,38), y "Teoría Probada"(27,29,32,35,37,39), sin embargo, el diseño metodológico utilizado no parece ser completamente adecuado para dicho fin.

En suma, se resalta la necesidad de hacer estudios con un diseño metodológico más robusto (por ejemplo, RCT para este tipo de estudios, teniendo en cuenta el marco de referencia $\mathrm{RE}_{-} \mathrm{AIM}^{(43)}$ ) que permitan medir la efectividad de una intervención en población de pacientes con cáncer de mama. Se sugiere que, para realizar estos estudios, se debe escoger una muestra representativa de la población ${ }^{(43)}$, con una distribución aleatoria -y no por conveniencia- a un grupo control y otro experimental, para garantizar la generalización de los resultados. Así mismo, se recomienda que la medición de la efectividad de lo anterior se realice a través de medida pre y post tratamiento, así como la importancia de tomar medidas durante la intervención con el fin de identificar la funcionalidad de cada módulo de trabajo y realizar las modificaciones pertinentes para futuras investigaciones. De igual manera se sugiere realizar medidas de seguimiento posterior por lo menos a los seis meses de finalizada la intervención ${ }^{(43)}$, con el fin de evaluar la sostenibilidad de los resultados a través del tiempo.

Adicionalmente se sugiere que se especifique el tamaño del efecto de estas intervenciones en las medidas correspondientes, utilizando el estadístico $d$ de Cohen para mayor precisión. Por último, y teniendo en cuenta que es difícil lograr obtener una muestra de la población totalmente aleatorizada y controlar todas las variables, se recomienda en los casos en que lo anterior no sea factible, realizar el análisis de resultados a través de una taxonomía de modelos por medio de regresiones, para identificar si los cambios que se presentan en las variables estudio, son realmente producto de la intervención. 


\subsection{Limitaciones de este estudio}

En cuanto a las limitaciones de este estudio, se encontró una dificultad al realizar la primera búsqueda en otras bases de datos, planeadas inicialmente y no tenidas en cuenta para este informe, de las cuales no se pudo obtener información. De igual manera, los pocos resultados que se encontraron en la primera búsqueda, derivados de la poca evidencia existente de intervenciones psicológicas en pacientes con cáncer de mama en Latinoamérica, implicaron una limitante para el objetivo de esta investigación, por lo que fue necesario ampliar la ventana de observación de artículos a España, y al año de publicación de la investigación.

\subsection{Futuras investigaciones}

Los resultados de esta revisión resaltan la necesidad de realizar mayor número de investigaciones de diseño robusto sobre las intervenciones psicológicas en la población de cáncer de mama, tanto en Latinoamérica y España, como a nivel mundial. Así mismo se resalta la necesidad de que los estudios realizados en población latinoamericana y española, así como su aplicación en la realidad de la psicología clínica y de la salud, esté relacionada con la evidencia que se presenta sobre las intervenciones a nivel mundial sobre esta población.

\section{Agradecimientos}

Esta investigación se realizó gracias al apoyo y financiación de ColcienciasColfuturo, en el marco de la Beca Para la Formación de Capital Humano de Alto Nivel para las Regiones - Departamento del Atlántico - Maestría Nacional - 673-, que se llevó a cabo en la Universidad de los Andes, Bogotá - Colombia, bajo el programa de Maestría en Psicología Clínica y de la Salud.

\section{Referencias bibliográficas}

1. Organización Mundial de la Salud (OMS). Enfermedades no Transmisibles. 2015. [Acceso 15 de enero de 2018]. Disponible en: http://www.who.int/mediacentre/factsheets/fs355/es/

2. Organización Mundial de la Salud (OMS). Cáncer. 2015. [Acceso Septiembre de 2016]. Disponible en: http://www.who.int/mediacentre/factsheets/fs297/es/

3. Cazap E, Buzaid A, Garbino C, Garza J, Orlandi F, Schwartsmann, et al. Breast cancer in Latin America Results of the Latin America and Caribbean Society of Medical Oncology/ Breast Cancer Research Foundation Expert Survey. Cancer Suppl 2008;113, 8,2359-65. doi $10.1002 /$ cncr.23834

4. Justo N, Wilking N, Jonsson B, Lucciani S, Gazap E. A review of breast cancer care and outcomes in Latin America. Oncologist 2013;18:248-56. doi: 10.1634/ theoncologist.2012-0373

5. Lozano-Ascencio R, Gómez-Dantés H, Lewis S, Torres-Sanchez L, López-Carrillo L. Tendencias del cáncer de mama en América Latina y el Caribe. Salud Publica Mex 2009;51:S147-S156

6. Bearison D, Pacifici C. Psychological studies of children who have cancer. J Appl Dev Psycho 1984;5:263-80 
7. Irvine D, Brown B, Crooks D. Psychosocial adjustment in women with breast cancer. Cancer 1991;67:1097-117

8. Burgess C, Cornelius V, Love S, Graham J, Richards M, Ramirez A. Depression and anxiety in women with early breast cancer five years observational cohort study. Brit Med J 2005;330:702-5. doi: 10.1136/bmj.38343.670868.D3

9. Tjemsland L, Soreide J, Malt U. Posttraumatic distress symptoms in operable breast cancer III: status one year after surgery. Breast Cancer Res Treat 1998;47:141-51.

10. Vassard D, Olsen M, Zinckernagel L, Vibe-Petersen J, Oksbjerg S, Johansen C. Psychological consequences of lymphoedema associated with breast cancer: A prospective cohort study. Eur J Cancer 2010;46:3211-8. doi: 10.1016/j.ejca.2010.07.041

11. American Psychological Association (APA). Cómo puede la mente ayudar al cuerpo. [Acceso el 10 de enero de 2018]. Disponible en: http://www.apAorg/centrodeapoyo/ cancer-de-mamAaspx

12. Robles R. Manejo de enfermedades crónicas y terminales. En Oblitas L, editor. Psicología de la Salud y Calidad de Vida Mexico: CENGAGE Learning 2010: 249-77

13. Monsalve V, Gómez-Carretero P, Soriano J. Intervención psicológica en dolor oncológico: un estudio de revisión. Psicooncología 2006;3:139-52

14. Bragado. C. Funcionamiento psicosocial e intervenciones psicológicas en niños con cáncer. Psicooncología 2009;6:327-41

15. Salas M, Gabaldón O, Mayoral JL, Guerrero R, Albisu J, Amayra I. Effective psychological interventions for coping with painful medical procedures in pediatric oncology: theoretical review. An Pediatr (Barc) 2003;59:41-7. doi: 10.1016/S1695-4033(03)78146-6

16. Bellver A, Moreno P. Riesgos psicosociales e intervención psicológica en los pacientes trasplantados de medula ósea. Psicooncología 2009;6:65-81

17. Pietro A. Psicología Oncológica. Revista Profesional Española de Terapia CognitivoConductual 2004;2:107-20

18. Matsuda A, Yamaoka K, Tango T, Matsuda T, Nishimoto. H. Effectiveness of psychoeducational support on quality of life in early-stage breast cancer patient: a systematic review and meta-analysis of randomized controlled trials. Qual Life Res 2014;23:21-30. doi: 10.1007/s11136-013-0460-3

19. Font. A., Rodriguez. E. Eficacia de las intervenciones psicológicas en mujeres con cáncer de mama. Psicooncología 2007;4:423-46.

20. McGregor B, Antoni M. Psychological intervention and health outcomes among women treated for breast cancer: a review of stress pathways and biological mediators. Brain Behav Immun 2008. doi:10.1016/j.bbi.2008.08.002

21. Kangas M, Bovbjerg D, Montgomery G. Cancer-related Fatigue: A systematic and meta-analysis review of non-pharmacological therapies for cancer patient. Psychol Bull 2008;134:700-41. doi: 10.1037/a0012825

22. Smith J, Richardson J, Hoffman C, Pilkington K. Mindfulness-Based Stress Reduction as supportive therapy in cancer care: systematic review. J Adv Nurs 2005;52:315-27.

23. Casellas-Grau A, Font A, Vives J. Positive Psychology interventions in breast cancer. A systematic review. Psychooncology 2014;23:9-19. doi: 10.1002/pon.3353

24. Telles D, Rodríguez A, Da Silva F, Luz C, Ferreira A, Campio M, et al. Relaxation and guided imagery program in patients with breast cancer undergoing radiotherapy is not associated with neuroimmunomodulatory effects. J Psychosom Res 2007;63:647-55.

25. Rodríguez E, Font A Eficacia de la terapia de grupo en cáncer de mama: Evolución de las emociones desadaptativas. Psicooncología 2013;10:275-87. doi: 10.5209/rev_PSIC.2013. v10.n2-3.43449 
26. Pocino M, Luna G, Canelones P, Mendoza A, Romero G, Palacios L, et al. La relevancia de la intervención psicosocial en pacientes con cáncer de mama. Psicooncología 2007;4:59-73.

27. Páez M, Luciano C, Guitiérrez O. Tratamiento psicológico para el afrontamiento del cáncer de mama. Estudio comparativo entre estrategias de aceptación y de control cognitivo. Psicooncología 2007;4:75-95.

28. Narváez A, Rubiños C, Cortés-Funes F, Gómez R, García A. Valoración de la eficacia de una terapia grupal cognitivo-conductual en la imagen corporal, autoestima, sexualidad y malestar emocional (ansiedad y depresión) en pacientes de cáncer de mama. Psicooncología 2008;5:93-102.

29. Montesinos F, Luciano C. Acceptanse of relapse fears in breast cancer patients: Effects of an ACT-based abridged intervention. Psicooncologia 2016;13:7-21. doi: 10.5209/rev_ PSIC.2016.v13.n1.52484

30. Manos D, Sebastian J, Mateo N, Bueno M. Results of a multi-componential psychosocial intervention programme for women with early-stage breast cancer in Spain: Quality of life and mental adjustment. Eur J Cancer 2008;18:295-305. doi: 10.1111/j.13652354.2008.00978.x

31. Lugo I, Becerra A, Reynoso L. Intervención psicológica para manejo de ansiedad ante la colocación de catéter puerto en mujeres con cáncer de mama. Psicooncología 2013;10:177-84. doi: 10.5209/rev_PSIC.2013.v10.41956

32. Gonzalez A, González. A, Estrada B. Eficacia de la terapia cognitivo conductual en mujeres con cáncer de mama. Psicooncología 2015;12:129-40. doi: 10.5209/rev_PSIC.2015.v12. n1.48908

33. García F, Rincón P. Prevención de Sintomatología Postraumática en Mujeres con Cáncer de Mama: Un modelo de intervención narrativo. Ter Psicol 2011;29:175-83.

34. Gallardo I, Durán L, Marrero Y. Eye Movement Desensitization and Reprocessing, método psicoterapéutico para disminuir la ansiedad en mujeres, antes de la mastectomía. Correo Científico Médico 2011;15(1).

35. Cerezo M, Ortiz-Tallo M, Cardenal V, Torre-Luque A. Positive psychology group intervention for breast cancer patients: A randomized trial. Psychol Rep 2014;115:44-64. doi 10.2466/15.20.PR0.115c17z7

36. Cerezo M, Ortiz-Tallo M, Cardenal V. Expresión de emociones y bienestar en un grupo de mujeres con cáncer de mama: una intervención psicológica. Rev Latinoam Psicol 2009;41:131-40.

37. Bernal L, Téllez A, Juárez D, García C, García E. El efecto de la hipnoterapia en la calidad de sueño de mujeres con cáncer de mama. Psicooncologia 2015;12:39-49. doi: 10.5209/rev_PSIC.2015.v12.n1.48902

38. Bellver A, Sanchez-Canovas J, Santaballa A, Munárriz B, Pérez-Fidalgo J, Montalar J. Mujeres con cáncer de mama: Evaluación del afecto positivo y negativo y valoración de un programa de intervención psicológica en el ámbito hospitalario. Psicooncología 2009;6:139-54.

39. Bellver A. Eficacia de la terapia grupal en la calidad de vida y el estado emocional en mujeres con cáncer de mama. Psicooncología 2007;4:133-42.

40. Araújo A, Desidéiro M, Diaz L, Duarte S, Sales J, Chada E. The biopsychosocial spiritual model applied to the treatment of women with breast cancer, throught RIME intervention (relaxation, mental images, spirituality). Complement Ther Clin Pract 2015;21:1-6. doi: 10.1016/j.ctcp.2015.01.007 
41. Glanz K, Bishop D. The Role of behavioral science theory in development and implementation of public health intervention. Ann Rev Public Health 2010;31:399-418. doi: 10.1146/annurev.publhealth.012809.103604

42. Barlow DH, Farchione T J, Fairholme CP, Ellard KK, Boisseau CL, Allen LB, et al. Unified protocol for transdiagnostic treatment of emotional disorders: Therapist guide. USA: Oxford University Press, 2011.

43. Glasgow R, Linnan L. Evaluation of theory-based intervention. Glanz K, Rimer B, Viswanath K. editors. Health behavior and health education. theory, research and practice (4a Ed) San Francisco, EE.UU: Wiley \& Sons, 2008. p. 487-508. 